\title{
Using Probabilistic Classification Technique and Statistical Features for Brain Magnetic Resonance Imaging (MRI) Classification: An Application of AI Technique in Bio-Science
}

\author{
Fazli Wahid ${ }^{*}$, Rozaida Ghazali ${ }^{2}$, Muhammad Fayaz $^{3}$ and Abdul Salam Shah ${ }^{4}$ \\ ${ }^{1,2}$ Universiti Tun Hussein Onn Malaysia \\ ${ }^{3}$ University of Malakand, KPK, Pakistan \\ ${ }^{4}$ SZABIST, Islamabad, Pakistan \\ ${ }^{\text {*w}}$ wahid_uomian@hotmail.com, ${ }^{2}$ rozaida@uthm.edu.my, \\ 33amaz_khan@yahoo.com, ${ }^{4}$ shahsalamss@gmail.com
}

\begin{abstract}
There are many medical imaging modalities used for the analysis and cure of various diseases. One of the most important of these modalities is Magnetic Resonance Imaging (MRI). MRI is advantageous over other modalities due to its high spatial resolution and the excellent capability of discrimination of soft tissues. In this paper, an automated classification approach of normal and pathological MRI is proposed. The proposed model three simple stages; preprocessing, feature extraction and classification. Two types of features; color moments and texture features have been considered as main features for the description of brain MRI. A probabilistic classifier based on logistic function has been used for the MRI classification. A standard data set consisting of one hundred and fifty images has been used in the experiments, which was divided into 66\% training and $34 \%$ testing. The proposed approach gave $98 \%$ accurate results for training data set and $94 \%$ accurate results for the testing data set. For validation of the proposed approach, 10Fold cross validation was applied, which gave $90.66 \%$ accurate results. The classification capability of probabilistic classifier has been compared with the different state of art classifiers, including Support Vector Machine (SVM), Naïve Bayes, Artificial Neural Network (ANN), and Normal densities based linear classifier.
\end{abstract}

Keywords: Brain MRI, Classification, Color Features, Probabilistic Classifier, Pathological MRI, Texture Features

\section{Introduction}

Many imaging modalities exist for the diagnosis and treatment of different abnormalities in the human body. For the diagnosis of the abnormalities of the human brain, Magnetic Resonance Imaging (MRI) and Computed Tomography (CT) are used. The capability of discrimination of soft tissues and high spatial resolution of MRI makes it advantageous over CT and other imaging techniques. Since MRI is very efficient in providing detailed and finer description of human brain tissues, it is mostly preferred by medical experts for diagnostic purpose as well as researchoriented activities [1].

The rich information provided by MRI about the human brain soft tissues has brought dramatic improvement in both qualitative and quantitative analysis of human brain structure [2]. This information also assists other imaging modalities like Positron Emission Tomography (PET) in their functional performance [3]. Radiologist experts perform sophisticated process for the assessment of features of MRI and characterization of different diseases in the human brain. The radiologists have achieved a high degree of precession and accuracy with those processes. 
Nevertheless, for bringing more effectiveness in the treatment, medical experts investigate different imaging techniques to achieve more accuracy in the pathological characterization of the soft tissues of the human brain [4].

The primary source of investigation for finding any pathological characterization in the human brain is the visual interpretation of the film. Almost all human body functions such as blood pressure, memory, emotions, temperature, heartbeat and fluid balance are under the direct or indirect control of the human brain [5]. Discrimination between the normal and pathological brain MRI is, therefore, critically important. Usually, we need a large number of images to compare different human brains and determine their difference [6-7]. As the authors in [8] compared 176 healthy brain images to find their differences, whereas in [9] collected 500 pediatric human brain images to study their characteristics.

The increase in the number of images for studying the pathological characterization of the human brain, a higher accuracy can be achieved. But, there are many limitations in manual inspection such as lack of reproducibility, long processing time and inaccuracies in the diagnosis. In order to achieve more accuracy in diagnosis, improve the quality of treatment and reduce the computation time, many automatic algorithms have been proposed and developed [10-13]. The shortage of expert radiologists and the increasing cost of manual inspection make the process more and more difficult. This gives a call for automating the process for the classification of normal and abnormal human body parts. If an image is a double read, it gives the better analysis.

The remaining structure of the paper is, section 2 presents Related Work, section 3 contains Proposed Methodology, section 4 contains Results and Discussion and finally, in section 5, Conclusion and Future Work is provided.

\section{Related Work}

The standard methodology for the classification of normal and pathological human brain MRI consists of four stages; preprocessing, feature extraction, dimensionality reduction, and MRI classification. The methodology is summarized in the Figure 1.

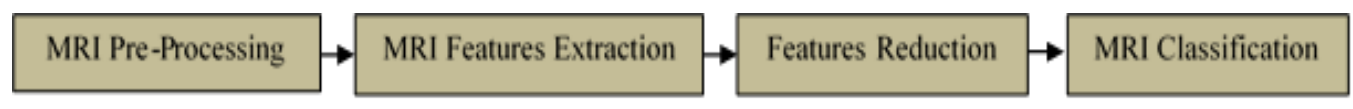

Figure 1: Brain MRI Classification Methodology

The authors in [14] proposed an approach for brain MRI processing and segmentation. The main step towards brain MRI segmentation was the estimation of features which was based on the correlation between the brain tumor and Lateral Ventricular (LV) and then these features were used for differentiation of different brain parts. The whole process of segmentation was accomplished in four stages; Pre-processing, Feature extraction, Segmentation, and Classification.

In the pre-processing stage, the information irrelevant for brain MRI processing that increases the computation time and complexity was addressed. In this stage, data redundancy, non-uniformity in the geometry of images, non-standardization in the intensity of images and the skull removal are addressed. LV has been used for the extraction of informative features. For the brain segmentation, Fuzzy Connected C-Mean (FCM) has been used whereas for classification, K-Nearest Neighbor has been applied.

The authors in [15] presented an approach for binary classification of the benign and malignant brain tumor. In the proposed approach, Wavelet Co-Occurrence Texture (WCT) and Wavelet Statistical Features (WST) has been combined for the 
binary classification. The process of classification has been carried out in six states; segmentation of the Region of Interest (ROI), two level decomposition of Discrete Wavelet (DW), Feature Extraction, Feature Selection, Classification and Performance Evaluation. For the brain, MRI segmentation, the Support Vector Machine (SVM) has been applied. For feature extraction, WCT and WST were used. For the selection of optimal features, Genetic Algorithm (GA) was applied. For the classification of the benign and malignant tumor, Probabilistic Neural Network (PNN) was applied.

The authors in [16] introduced a new method for the binary classification of brain MRI into normal and abnormal. The proposed method consists of three stages; Feature Extraction, Feature Reduction, and Classification. For the feature extraction, Two- Dimensional Discrete Wavelet Transform (2D DWT) has been applied. For feature reduction, Principal Component Analysis (PCA) and Linear Discriminant Analysis (LDA) were used. In the last stage, the extracted features were fed to Support Vector Machine (SVM) and k-Nearest Neighbor (k-NN) for discrimination of normal and abnormal MRI. They have claimed higher classification accuracy with using less number of features as compared to other well-known approaches applied in the literature.

The authors in [17], applied Support Vector Machine (SVM) for brain MRI classification. For descriptive feature extraction, the spectral angle based approach was used. For the division of MRI into different regions, distance based clustering was applied. For feature reduction, Independent Component Analysis (ICA) was applied, and finally, the MRI's were classified into normal and abnormal using Support Vector Machine (SVM).

The authors in [18], proposed two steps approach for brain MRI classification. In the first stage, the informative features were extracted from brain MRI using the spider web plot which is based on wavelet entropy. For classification of MRI into normal and abnormal, Probabilistic Neural Network (PNN) was applied.

The authors in [19] used an Artificial Neural Network (ANN) for classification of normal and abnormal (benign and malignant) MRI. The approach consists of three stages; Feature Extraction, Feature Reduction, and finally Classification. In order to extract features from MRI, Principal Component Analysis (PCA) was used. The important features extracted from brain MRI were mean, variance, and median, correlation values of minimum and maximum intensity. For the classification of MRI into normal, a benign tumor and malignant tumor, the Back Propagation Artificial Neural Network (BP-ANN) was designed. The BP-ANN was proved as an excellent classifier for accurate and fast classification.

The authors in [20] introduced a novel approach for finding any abnormality in different brain parts. The proposed approach was applied to different types of imaging modalities including T2 weighted images, T1 weighted images, PD images and MRI images. The developed approach consists of four main stages; Image PreProcessing, Feature Extraction, Feature Reduction, and Classification. In the first stage, the increase in contrast between the abnormal and normal tissues of brain images is carried out and the Discrete Fourier Transform (DFT) of the image is calculated. In the proposed approach non-statistical features have been extracted from the images and kernel-F score method has been used for feature reduction. These reduced features are fed to Multi-Layer Perceptron (MLP) for classification.

For the classification of normal and abnormal brain MRI, the authors in [21] applied Artificial Neural Network (ANN). The task of classification was accomplished in three stages; Pre-Processing, Feature Extraction and Classification. For sharpening and enhancement of brain MRI images, Morphological Operations, and Histogram Equalization were performed in the pre-processing stage. For 
extraction of valuable features, Grey Level Co-occurrence Matrix (GLCM) was applied.

A hybrid technique was used by the authors in [22] for brain MRI classification. The classification process has been carried out in three stages; Feature Extraction, Feature Reduction, and MRI Classification. Discrete Wavelet Transform (DWT) has been used for feature extraction. For feature reduction, Principal Component Analysis (PCA) has been used. In the classification stage, two classifiers; k-Nearest Neighbor (k-NN) and Feed Forward Back Propagation Artificial Neural Network FP-ANN) have been used for binary classification (normal and abnormal MRI). The results obtained have been compared with the state of art techniques and found effective and robust in comparison with the other approaches.

For discrimination of different types of brain tumors, the authors in [23] proposed a software application. The approach was applied to T1 weighted images. For feature extraction, Least Squared Feature Transformation (LSFT) was applied whereas for classification, a modified form of Probabilistic Artificial Neural Network (PANN) was used. The method was applied to discriminate the primary and secondary brain tumor and also to discriminate the gliomas and meningiomas.

\section{Proposed Methodology}

The proposed methodology performs the classification of normal and pathological MRI in three stages as described in Figure 2.

- $\quad$ MRI Pre-Processing.

- Features Extraction.

- $\quad$ MRI Classification.

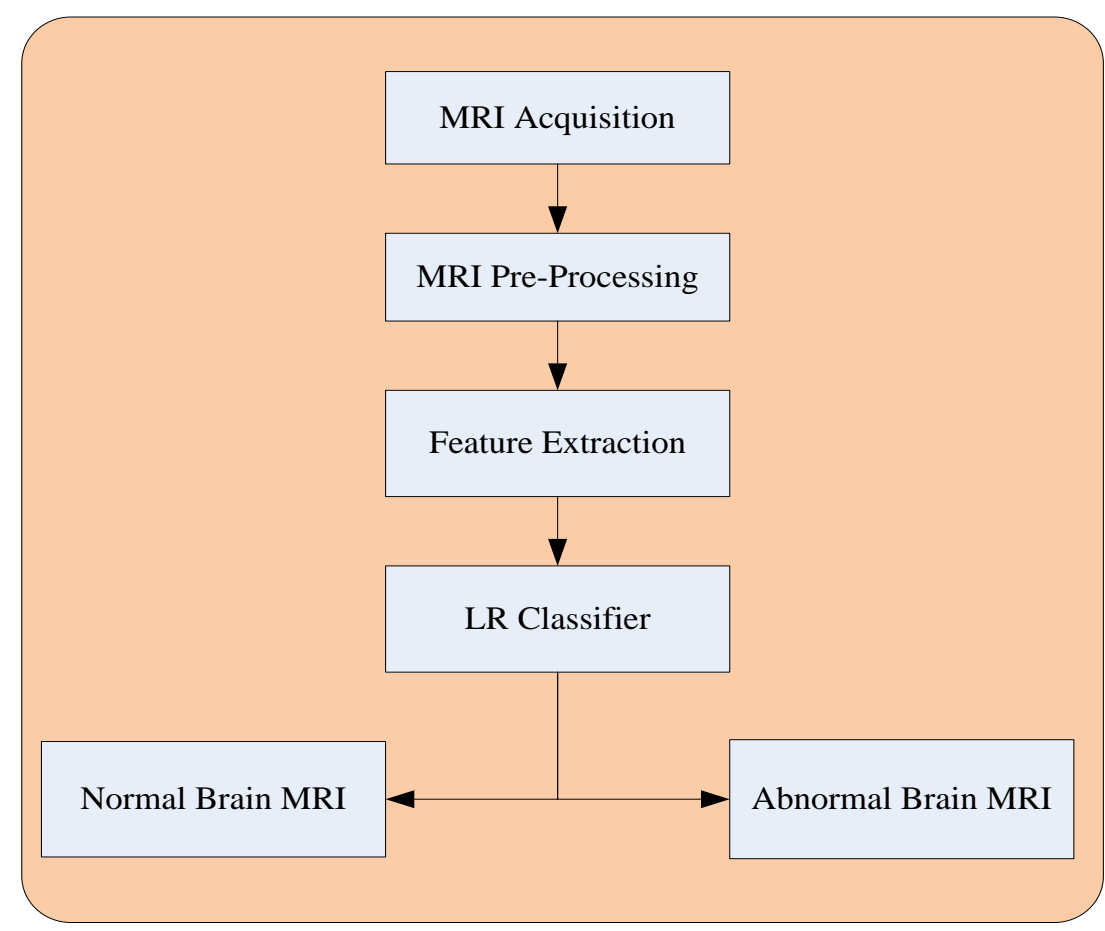

Figure 2. Proposed Model

\subsection{Data Set Description}

There are different types of diseases that affect the structure of the brain and cause changes in the intensities of pixels representing different human brain parts. 
In this paper, we have considered four types of brain MRI for processing; normal MRI, MRI affected by acute stroke, MRI affected by Alzheimer disease and MRI affected by the tumor. We have a total of $150 \mathrm{~T} 2$ weighted images downloaded from www.med.harvard.edu/AANLIB (Harvard Medical School Website). Out of these 150 images, 75 were normal and 75 were abnormal images. The abnormal brain images including 25 images affected by acute stroke, 25 affected by Alzheimer disease and 25 general tumor images. Figure 3 shows one image from each of these four classes. During experimentation, we have performed the binary classification of brain MRI into normal an abnormal MRI. 75 normal images have been put in one class and other 75 images ( 25 images of each disease) have been put in the other class.
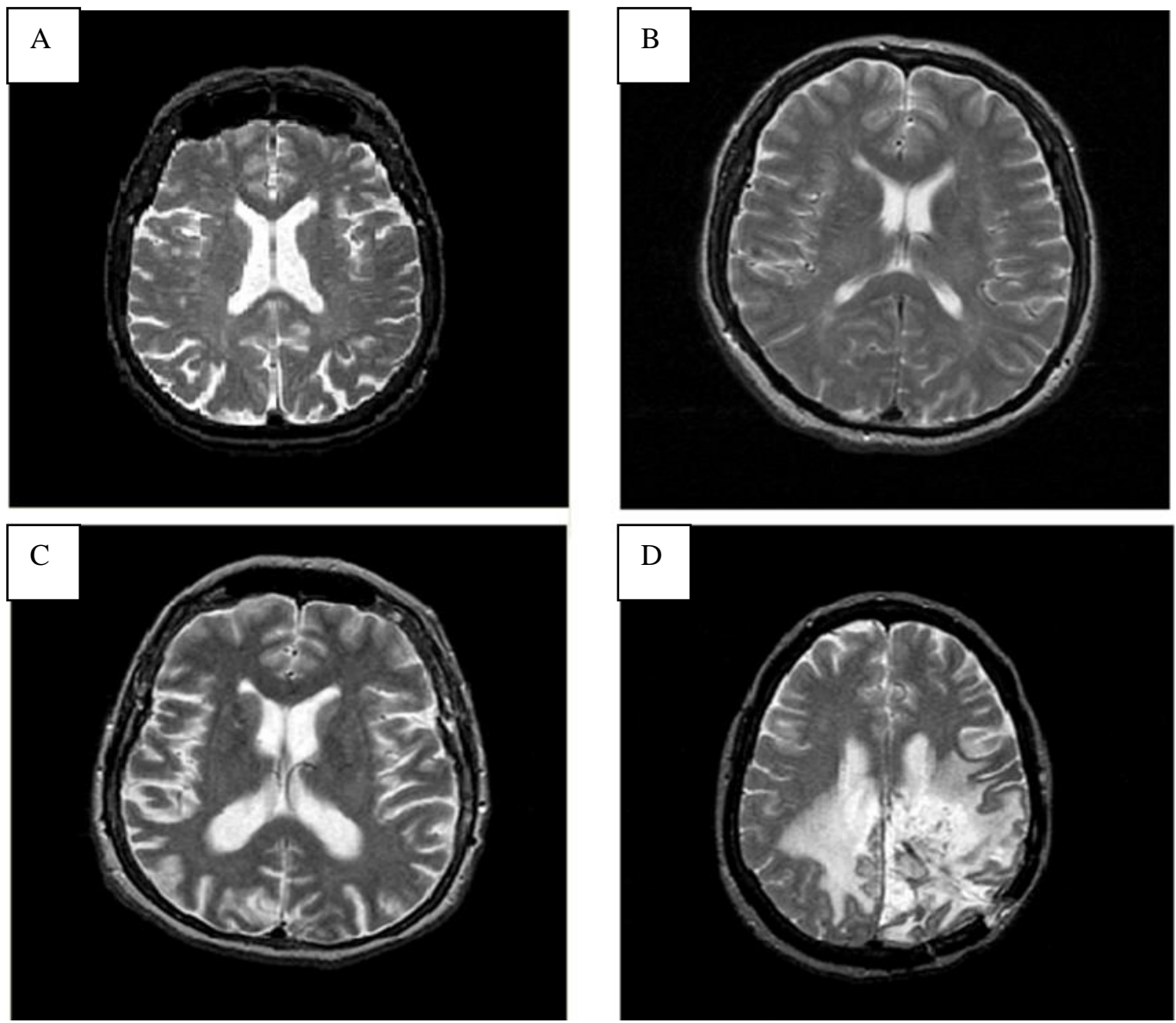

Figure 3. A. Normal MRI, B. MRI with Acute Stroke, C. MRI with Alzheimer Disease, D. MRI with Tumor

\subsection{MRI Pre-Processing}

After the image acquisition the pre-processing step is applied for removal of the background and noise from the brain MRI. At this stage, some undesired components such as scalp and skull are also removed. Mainly three activities are performed during this step; skull removal, noise removal and the transformation of MRI to some other suitable format for further processing. The main type of noise that affects the MRI is the salt and pepper noise. The median filter is considered to be the best option for removal of salt and pepper noise [24]. The median filter has the capability of removal of noise without disturbing the edges of the image. In the proposed model, a median filter of size $3 \times 3$ has been applied. After noise removal, 
mostly the skull is removed from the brain MRI which contains some components which do not represent the actual brain [25].

Actually, the brain constitutes a small portion of the whole brain MRI. There are many extra parts like the skull itself and the air present inside the MRI. There are standard data sets available with the skull already removed for enabling researchers to carry out the task very easily [26]. For this study the images have been downloaded from the Harvard Medical College website with the skull already removed. During the image transformation stage, the MRI is converted from grey scale to color MRI for extraction of color moments which are the main features on the basis of which the classification of brain MRI will be performed. We will extract three color (Red, Green and Blue) channels from the image to extract RGB features. Figure 4 (a) shows the grey scale image whereas figure 4 (b) represents the color (RGB) image after transformation.

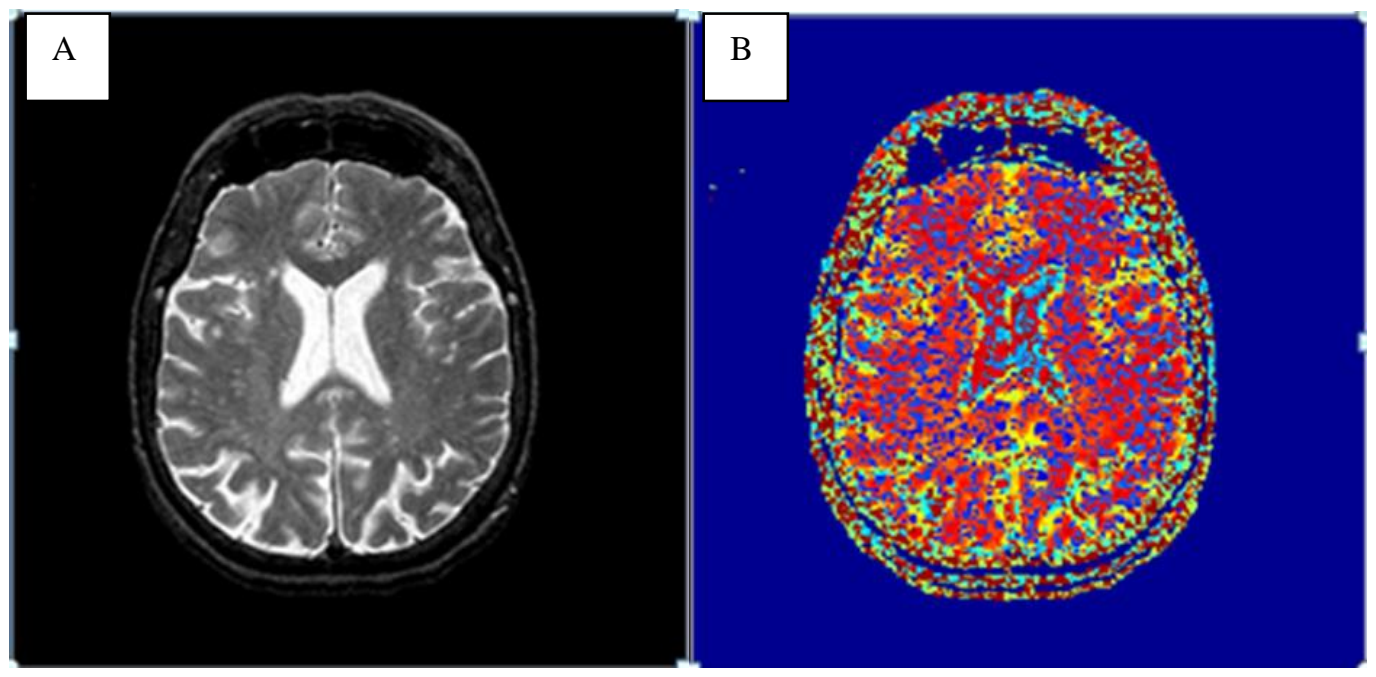

Figure 4. A. Grey scale MRI Image before the Pre-Processing B. Grey Scale MRI Image after Pre Processing

\subsection{MRI Feature Extraction}

In the proposed algorithm, two types of features have been considered.

1. Color Moments.

2. Texture Features.

Color moments and texture features are extracted from the color image after the conversion of the grey scale image to color MRI. For extraction of the color moments, the first three channels; Red, Blue and Green are separated from the color MRI and then extracted from the RGB image. First four color moments, i.e. Mean, Variance, Skewness, and Kurtosis are computed for each of the three color channels. Three texture features; Mean Absolute Deviation (MAD), Energy and Entropy have been considered for the MRI classification in the proposed technique. The detailed description of the extracted features from color MRI having four Color Features and three Texture Features, including their mathematical representation is provided in the Table. 1. 
Table 1. Extracted Features

\begin{tabular}{|c|c|c|}
\hline \multicolumn{3}{|c|}{ Color Moments } \\
\hline Feature & Equation & Description \\
\hline Mean & $M=\frac{1}{N} \sum_{i=1}^{N} x_{i}$ & $\begin{array}{l}\text { Mean represents the average of intensities } \\
\text { of all pixels of the red, green and blue } \\
\text { channel. }\end{array}$ \\
\hline Variance & $V=\frac{1}{N} \sum_{i=1}^{Y}\left(x_{i}-M\right)^{2}$ & $\begin{array}{l}\text { Variance represents the variations in the } \\
\text { intensities of all pixels of the red, green } \\
\text { and blue channel. }\end{array}$ \\
\hline Skewness & $S=\frac{1}{N} \sum_{i=1}^{W}\left(x_{i}-M\right)^{a}$ & $\begin{array}{l}\text { Skewness represents the asymmetry in the } \\
\text { intensities of all pixels of the red, green } \\
\text { and blue channel. }\end{array}$ \\
\hline Kurtosis & $K=\frac{1}{M} \sum_{i=1}^{W}\left(x_{i}-M\right)^{4}$ & $\begin{array}{l}\text { It represents the peak or frequency of the } \\
\text { extreme in the intensities of all pixels of } \\
\text { the red, green and blue channel. }\end{array}$ \\
\hline \multicolumn{3}{|c|}{ Texture Features } \\
\hline $\begin{array}{c}\text { Mean } \\
\text { Absolute } \\
\text { Deviation }\end{array}$ & $\mathrm{MD}=\frac{1}{N} \sum_{i=1}^{N}\left|x_{i}-M\right|$ & $\begin{array}{l}\text { Represents the mean of absolute deviation } \\
\text { pixel intensities in each channel. }\end{array}$ \\
\hline Energy & $\mathrm{EN}=\frac{1}{N} \sum_{i=1}^{N}\left|x_{i}\right|^{2}$ & $\begin{array}{l}\text { Represents the amount of variations in the } \\
\text { pixel intensities in each channel. }\end{array}$ \\
\hline Entropy & $\mathrm{ENT}=-\sum_{i} x_{i}{ }^{2} \log x_{i}^{2}$ & $\begin{array}{l}\text { Represents the randomness in the statistical } \\
\text { measurements of the pixel intensities. }\end{array}$ \\
\hline
\end{tabular}

\subsection{MRI Classification}

The logistic regression based probabilistic classifier has been used for the classification of MRI into normal or abnormal [27]. The logistic regression is suitable for two class classification problems like normal MRI and abnormal MRI. The two class classification problem is known as a binary classification problem and multi class classification, as multinomial regression. In the proposed technique, we have used it for binary classification. Two types of vectors are used in logistic regression known as an input vector and output vector. The input vectors represent the set of features used as input to the classifier whereas the output vector represents the output class for each set of inputs. In order to describe the output class of input feature vector, a probabilistic function known as the logistic function is use d by the logistic regression [27].

\subsubsection{Logistic Function}

The probabilistic classifier used in the proposed technique uses a logistic function to find the probability of the input vector that belongs to a specific class [28]. The logistic function is mathematically expressed by (1).

$$
\mathrm{F}(\mathrm{t})=\frac{e^{\mathrm{t}}}{e^{\mathrm{t}}+1}=\frac{1}{1+e^{-t}}
$$

Where $F(t)$ represents the logistic function, ' $t$ ' represents the function of input feature and ' $\mathrm{e}$ ' represents the exponential function. 
Suppose, ' $\mathrm{x}$ ' represents an input feature or combination of input features, the logistic function can be calculated by (2).

$$
\mathrm{F}(\mathrm{x})=\frac{1}{1+e^{-\left(\beta_{0}+\beta_{1} x\right)}}
$$

Where $\mathrm{F}(\mathrm{x})$ represents the probability of the output class that a particular input feature will belong to. $\beta_{0}$ Represents the regression intercept, and $\beta_{1} x$ represents the coefficient of regression.

If we have more input features, then $\beta_{1} x$ can be calculated by (3).

$$
\beta_{0}+\beta_{1} x_{1}+\beta_{2} x_{2}+\cdots+\beta_{m} x_{m}
$$

3.4.2. Logit: The logit is the inverse of the logistic function is calculated by (4).

$$
\mathrm{g}(\mathrm{x})=\ln \frac{F(x)}{1-F(x)}=\beta_{0}+\beta_{1} x
$$

3.4.3. Odd: The logistic regression predicts the odd which shows that a particular combination of input features will belong to some particular output class, and it can be expressed by (5).

$$
\frac{F(x)}{1-F(x)}=e^{\left(\beta_{0}+\beta_{1} x\right)}
$$

\section{Experimental Results and Discussion}

In order to apply pre-processing and feature extraction, we used MATLAB 7.6.0 and for classification, we applied Weka 3.7.10. Both of these applications were installed on Core i3 with $2.0 \mathrm{~GB}$ of RAM and $2.40 \mathrm{GHz}$ processor.

\subsection{Algorithm Accuracy}

A standard data set consisting of one hundred and fifty images has been used in the experiments, which was divided into $66 \%$ training and $33 \%$ testing. For the training of classifier 50 normal and 50 abnormal images have been considered. The normal images have been classified with $100 \%$ accuracy while the abnormal ima ges have been classified with $96 \%$ accuracy during training. The overall accuracy of $98 \%$ have been achieved with a total of 100 images during training. The detail is provided in the table 2 . The remaining 25 normal and 25 abnormal images have been considered for the testing. The normal images have been classified with $96 \%$ accuracy while the abnormal images have been classified with $92 \%$ accuracy during the testing. The overall accuracy of $94 \%$ have been achieved during the testing with a total of 50 images as shown in the Table 3. The results in Table 2-3 have been achieved with 1 Fold cross validation, in order to observe proper deviation in results we have performed 10 fold cross validation. The 10 fold cross validation has been performed with 75 normal and 75 abnormal images. The normal images have been classified with $89.33 \%$ accuracy while the abnormal images have been classified with $92 \%$ accuracy with 10 fold cross validation. The overall accuracy of $90.66 \%$ have been achieved during the 10 fold cross validation with a total of 150 images as shown in the table 4 . The experimental results are quite satisfactory, but still can be improved. 
Table 2. Training Data Set Accuracy

\begin{tabular}{|c|c|c|c|}
\hline \multicolumn{4}{|c|}{ Classification Accuracy with Normal Images } \\
\hline Total Images & $\begin{array}{c}\text { Correctly Classified } \\
\text { Images }\end{array}$ & $\begin{array}{c}\text { Incorrectly Classified } \\
\text { Images }\end{array}$ & $\begin{array}{c}\text { Classification } \\
\text { Accuracy }\end{array}$ \\
\hline $\mathbf{5 0}$ & 50 & 0 & $100 \%$ \\
\hline \multicolumn{4}{|c|}{ Classification Accuracy with Abnormal Images } \\
\hline Total Images & $\begin{array}{c}\text { Correctly Classified } \\
\text { Images }\end{array}$ & $\begin{array}{c}\text { Incorrectly Classified } \\
\text { Images }\end{array}$ & $\begin{array}{c}\text { Classification } \\
\text { Accuracy }\end{array}$ \\
\hline $\mathbf{5 0}$ & 48 & 2 & $96.00 \%$ \\
\hline Overall Accuracy \\
\hline Total Images & $\begin{array}{c}\text { Correctly Classified } \\
\text { Images }\end{array}$ & $\begin{array}{c}\text { Incorrectly Classified } \\
\text { Images }\end{array}$ & $\begin{array}{c}\text { Classification } \\
\text { Accuracy }\end{array}$ \\
\hline $\mathbf{1 0 0}$ & 98 & 2 & $98.00 \%$ \\
\hline
\end{tabular}

Table 3. Testing Data Set Accuracy

\begin{tabular}{|c|c|c|c|}
\hline \multicolumn{4}{|c|}{ Classification Accuracy with Normal Images } \\
\hline Total Images & $\begin{array}{c}\text { Correctly Classified } \\
\text { Images }\end{array}$ & $\begin{array}{c}\text { Incorrectly Classified } \\
\text { Images }\end{array}$ & $\begin{array}{c}\text { Classification } \\
\text { Accuracy }\end{array}$ \\
\hline $\mathbf{2 5}$ & 24 & 1 & $96.00 \%$ \\
\hline \multicolumn{4}{|c|}{ Classification Accuracy with Abnormal Images } \\
\hline Total Images & $\begin{array}{c}\text { Correctly Classified } \\
\text { Images }\end{array}$ & $\begin{array}{c}\text { Incorrectly Classified } \\
\text { Images }\end{array}$ & $\begin{array}{c}\text { Classification } \\
\text { Accuracy }\end{array}$ \\
\hline $\mathbf{2 5}$ & 23 & 2 & $92.00 \%$ \\
\hline Total Images & $\begin{array}{c}\text { Correctly Classified } \\
\text { Images }\end{array}$ & $\begin{array}{c}\text { Incorrectly Classified } \\
\text { Images }\end{array}$ & $\begin{array}{c}\text { Classification } \\
\text { Accuracy }\end{array}$ \\
\hline $\mathbf{5 0}$ & 47 & 3 & $94.00 \%$ \\
\hline
\end{tabular}

Table 4. 10 Fold Cross Validation Accuracy

\begin{tabular}{|c|c|c|c|}
\hline \multicolumn{5}{|c|}{ Classification Accuracy with Normal Images } \\
\hline Total Images & $\begin{array}{c}\text { Correctly Classified } \\
\text { Images }\end{array}$ & $\begin{array}{c}\text { Incorrectly Classified } \\
\text { Images }\end{array}$ & $\begin{array}{c}\text { Classification } \\
\text { Accuracy }\end{array}$ \\
\hline $\mathbf{4 5}$ & 67 & 8 & $89.33 \%$ \\
\hline \multicolumn{4}{|c|}{ Classification Accuracy with Abnormal Images } \\
\hline Total Images & $\begin{array}{c}\text { Correctly Classified } \\
\text { Images }\end{array}$ & $\begin{array}{c}\text { Incorrectly Classified } \\
\text { Images }\end{array}$ & $\begin{array}{c}\text { Classification } \\
\text { Accuracy }\end{array}$ \\
\hline $\mathbf{7 5}$ & 69 & 6 & $92.00 \%$ \\
\hline \multicolumn{4}{|c|}{ Overall Accuracy } \\
\hline Total Images & $\begin{array}{c}\text { Correctly Classified } \\
\text { Images }\end{array}$ & $\begin{array}{c}\text { Incorrectly Classified } \\
\text { Images }\end{array}$ & $\begin{array}{c}\text { Classification } \\
\text { Accuracy }\end{array}$ \\
\hline $\mathbf{1 5 0}$ & 136 & 14 & $90.66 \%$ \\
\hline
\end{tabular}

\subsection{Comparative Analysis}

In this section, the classification accuracy of probabilistic classifier has been compared with the results achieved by [23] with Normal Densities Based Linear (NDBL) classifier, Naïve Bayes Classifier, Support Vector Machine (SVM), and Artifical Neural Network (ANN). The authors in [23] applied Leave One Out (LOO) and External Cross Validation (ECV) for discrimination of the primary and secondary brain tumor using different techniques. The proposed technique has 
outperformed all these techniques by a notable margin. The classifier based comparison of the probabilistic classifier is provided in the Table. 5. The detailed comparison of the proposed technique with LOO and ECV methods is provided in the Table 6 , and 7 , respectively.

Table 5. Comparative Analysis of Probabilistic Classifier with other Classifiers

\begin{tabular}{|c|c|c|}
\hline Serial No & Name of Classifier & $\begin{array}{c}\text { Classification } \\
\text { Accuracy }\end{array}$ \\
\hline $\mathbf{1}$ & Normal Densities Based Linear (NDBL) classifier & $75.38 \%$ \\
\hline $\mathbf{2}$ & Naive Bayes (NB) classifier & $72.82 \%$ \\
\hline $\mathbf{3}$ & Support Vector Machine (SVM) classifier & $87.60 \%$ \\
\hline $\mathbf{4}$ & Artificial Neural Network (ANN) & $92.75 \%$ \\
\hline & Proposed Algorithm (Logistic Regression) & $\mathbf{9 4 . 0 0 \%}$ \\
\hline
\end{tabular}

Table 6. Classification Results for Discrimination of Primary and Secondary Tumors using LOO Method [23]

\begin{tabular}{|l|c|c|c|}
\hline Approach Applied & $\begin{array}{c}\text { Accuracy of } \\
\text { Primary Tumor }\end{array}$ & $\begin{array}{c}\text { Accuracy of } \\
\text { Secondary } \\
\text { Tumor }\end{array}$ & $\begin{array}{c}\text { Overall } \\
\text { Classification } \\
\text { Accuracy }\end{array}$ \\
\hline 1. PNN & $86.96 \%$ & $95.24 \%$ & $89.55 \%$ \\
\hline 2. Linear LSFT-PNN & $89.13 \%$ & $95.24 \%$ & $91.04 \%$ \\
\hline 3. SVM-RBF & $91.30 \%$ & $85.71 \%$ & $89.55 \%$ \\
\hline Proposed Approach & Normal (96\%) & Abnormal (92\%) & $94.00 \%$ \\
\hline
\end{tabular}

Table 7. Classification Results for Discrimination of Primary and Secondary Tumors using ECV Method [23]

\begin{tabular}{|l|c|c|c|}
\hline \multicolumn{1}{|c|}{ Approach Applied } & $\begin{array}{c}\text { Accuracy of } \\
\text { Primary Tumor }\end{array}$ & $\begin{array}{c}\text { Accuracy of } \\
\text { Secondary } \\
\text { Tumor }\end{array}$ & $\begin{array}{c}\text { Overall } \\
\text { Classification } \\
\text { Accuracy }\end{array}$ \\
\hline 1. PNN & $84.38 \%$ & $52.86 \%$ & $74.78 \%$ \\
\hline 2. Linear LSFT-PNN & $89.38 \%$ & $37.14 \%$ & $73.48 \%$ \\
\hline 3. SVM-RBF & $93.75 \%$ & $30.00 \%$ & $74.35 \%$ \\
\hline 4. ANN & $88.13 \%$ & $61.43 \%$ & $80.00 \%$ \\
\hline 5. Cubic LSFT-PNN & $81.25 \%$ & $71.43 \%$ & $78.26 \%$ \\
\hline Proposed Approach & Normal (96\%) & Abnormal (92\%) & $94.00 \%$ \\
\hline
\end{tabular}

\section{Conclusion and Future Work}

In this paper, a probabilistic classifier based approach has been used for the classification of human brain MRI into normal or abnormal classes. The algorithm consists of three simple stages; preprocessing, feature extraction and MRI classification. In the preprocessing, the median filter has been applied to remove salt and pepper noise. In feature extraction, color moments and texture features have been extracted as main features. For extraction of color features, the grey scale image has been converted into color MRI and then red, green and blue channels have been extracted. Four color moments; mean, variance, skewness and Kurtosis; and three texture features; mean absolute deviation, energy and entropy have been computed for each of the red, green and blue channels. The probabilistic classifier based on logistic regression has been used for classification. The algorithm gave 
98\% accurate classification results for training data set, $94 \%$ accurate results for the testing data set and $90.66 \%$ accurate results for 10 -Fold cross validation. This simple algorithm can be extended to other types of classification, which is left as future work.

\section{References}

[1] M. Shenton, R. Kikinis, F. Jolesz, et al., "Abnormalities of the Left Temporal Lobe and Thought Disorder in Schizophrenia”, N. Eng. J. Med, vol. 327, (1992), pp. 604-612.

[2] L.M. Fletcher-Heath, L.O. Hall, D.B. Goldgof, F.R. Murtagh, "Automatic Segmentation of NonEnhancing Brain Tumors in Magnetic Resonance Images”, Artif. Intell. Med, vol. 21, no. 1-3, (2001), pp. 43-63.

[3] D. Levin, X. Hu, and K. Tan, et al., "The brain: Three-Dimensional Display of MR and PET Images", Radiol., vol. 172, (1989), pp. 783-789.

[4] R.A. Lerski, K. Straughan, L.R. Schad, D. Boyce, S. Bluml, I. Zuna, "MR Image Texture Analysis-an Approach to Tissue Characterization", Magn. Reson. Imaging, vol. 11, no. 6, (1993), pp. 873-887.

[5] J. S. Kim, V. Singh, J. K. Lee, J. Lerch, Y. Ad-Dab'bagh, D. Mac-Donald, J. M. Lee, S. I. Kim, and A. C. Evans, "Automated 3-D Extraction and Evaluation of the Inner and Outer Cortical Surfaces using a Laplacian Map and Partial Volume Effect Classification", NeuroImage, vol. 27, no. 1, (2005), pp. 210 221.

[6] H. K. Khan, A.S. Shah and M. A. Khan, "Critical Evaluation of Frontal Image-Based Gender Classification Techniques", International Journal of Signal Processing, Image Processing and Pattern Recognition, vol. 9, no.10, (2016), pp. 313-324.

[7] Z. Ullah., M. Fayaz, and A. Iqbal, "Critical Analysis of Data Mining Techniques on Medical Data", International Journal of Modern Education and Computer Science, vol. 8, no. 2, (2016), 42-48.

[8] E. R. Sowell, B. S. Peterson, P. M. Thompson, S. E. Welcome, A.L. Henkenius, and A. W. Toga, "Mapping Cortical Change Across the Human Life Span", Nature Neurosci, vol. 6, (2003), pp. 309-315.

[9] A. C. Evans, "The NIH MRI Study of Normal Brain Development", NeuroImage, vol. 30, (2006), pp. 184-202.

[10] C. A. Cocosco, A. P. Zijdenbos, and A. C. Evans, "A fully Automatic and Robust Brain MRI Tissue Classification Method”, Med. Image Anal., vol. 7, no. 4, (2003), pp. 513-527.

[11] A.S. Shah, M. N. A. Khan, F. Subhan, M. Fayaz, A. Shah, "An Offline Signature Verification Technique using Pixels Intensity Levels", International Journal of Signal Processing, Image Processing and Pattern Recognition, vol. 9, no.8, (2016), pp. 205-222.

[12] J. Tohka, A. Zijdenbos, and A. Evans, "Fast and Robust Parameter Estimation for Statistical Partial Volume Models in Brain MRI", NeuroImage, vol. 23, no. 1, (2004), pp. 84-97.

[13] V. Singh, "Use of a Non-Stationary Markov Random Field in Brain Tissue Partial Volume Segmentation", M.S. thesis, Dept. Elect. Comput. Eng., McGill Univ., Montreal, QC, Canada, Feb. (2005).

[14] K. Xiao, A. L. Liang, H. B. Guan, A. E. Hassanien, "Extraction and Application of Deformation Based Feature in Medical Images", Neuro Computing, Image Feature Detection and Description, vol. 120, (2013), pp.177-184.

[15] A.P. Nanthagopal and R. Sukanesh, "Wavelet Statistical Feature Based Segmentation and Classification of Brain Computed Tomography Images”, IET Image Processing, vol. 7, no.1, (2013), pp. 25-32.

[16] H. Kalbkhani, M. G Shayesteh, B. Zali-vargahan "Robust Algorithm for Brain Magnetic Resonance Image Classification based on GARCH Variances Series", Biomedical Signal Processing and Control, vol. 8, no. 6, (2013), pp. $909-919$.

[17] S. Sindhumol, A. Kumar, K. Balakrishnan, "Spectral Clustering Independent Component Analysis for Tissue Classification from Brain MRI”, Biomedical Signal Processing and Control, vol.8, (2013), pp. 667-674.

[18] M. Saritha, K.P. Joseph, A. T. Mathew, "Classification of MRI Brain Images using Combined Wavelet Entropy based Spider Web Plot and Probabilistic Neural Network", Pattern Recognition Letters, vol. 34, no. 16, (2013), 2151-2156.

[19] N. Sumitra, R.K. Saxena, "Brain Tumor Classification Using Back Propagation Neural Network", International Journal of Image, Graphics and Signal Processing, vol.5, no.2, (2013), pp. 45-50.

[20] A. E. Lashkari, "A Neural Network Based Method For Brain Abnormality Detection in MR Images Using Gabor Wavelet", International Journal of computer Application, vol. 4, no. 7, (2010), pp.9-15.

[21] S. Jain, "Brain Classification Using GLCM Based Feature Extraction in Artificial Neural Network", International Journal of Computer Science \& Engineering Technology (IJCSET), vol. 4, no.7, (2013), pp.966-970.

[22] E. S. A. Ei-Dahshan, T. Hosny, A.B.M. Salem, "Hybrid Intelligent Technique for MRI Brain Image Classification", Digital Signal Processing, vol. 20, no.2, (2010), pp. 433-441.

[23] P. Georgiadis, D. Cavouras, I. Kalatizis, A. Daskalakis, G.C. Kagadis, K. Sifaki, M.Malamas, G. Nikifordis, and E.Solomou, "Improving Brain Tumor Characterization On MRI By Probabilistic 
Neural Network and Non Linear Transform of Texture Feature", Computer Methods and Program in Biomedicine, vol.89, no.1, (2008), pp. 24-32.

[24] M. Fayaz, A. S. Shah, F. Wahid, A. Shah, "A Robust Technique of Brain MRI Classification using Color Features and K-Nearest Neighbors Algorithm", International Journal of Signal Processing, Image Processing and Pattern Recognition, vol. 9, no.10, (2016), pp.11-20.

[25] F. Wahid, M. Fayaz, and A.S. Shah, "An Evaluation of Automated Tumor Detection Techniques of Brain Magnetic Resonance Imaging (MRI)”, International Journal of Bio-Science and Bio-Technology, vol. 8, no.2, (2016), pp.265-278.

[26] M. Nazir, F. Wahid and S. A. Khan, "A Simple and Intelligent Approach for Brain MRI Classification", Journal of Intelligent \& Fuzzy Systems: Applications in Engineering and Technology, vol. 28, no. 3, (2015), pp. 1127-1135.

[27] http://en.wikipedia.org/wiki/Logistic_regression.

[28] D.W. Hosmer, S. Lemeshow, Applied Logistic Regression, Second Edition, Wiley. ISBN 0-471-356328, (2005).
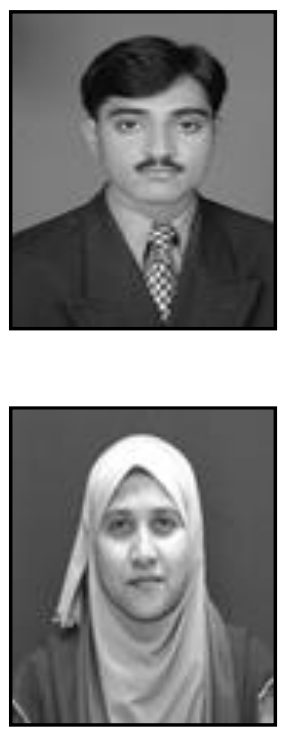

\section{Authors}

Fazli Wahid, he received BS in Computer Science from University of Malakand, Pakistan in 2006, and MS in Computer Science from SZABIST, Islamabad, Pakistan in 2015. He is currently with University Tun Hussein Onn Malaysia.

His areas of interest are energy consumption prediction, optimization, and management using multilayer perceptron, Artificial Bee Colony, Ant Colony, Swarm Intelligence, and other Machine Learning Techniques.

Rozaida Ghazali, she is currently an Associate Professor at the Faculty of Computer Science and Information Technology, Universiti Tun Hussein Onn Malaysia (UTHM). She graduated with a Ph.D. degree in Higher Order Neural Networks from the School of Computing and Mathematical Sciences at Liverpool John Moores University, United Kingdom in 2007. Earlier, in 2003 she completed her M.Sc. degree in Computer Science from Universiti Teknologi Malaysia (UTM). She received her B.Sc. (Hons) degree in Computer Science from Universiti Sains Malaysia (USM) in 1997. In 2001, Rozaida joined the academic staff in UTHM. Her research area includes neural networks, swarm intelligence, optimization, data mining, time series prediction, and data classification. She has successfully supervised a number of $\mathrm{PhD}$ and master students and published more than 100 articles in various international journals and conference proceedings. She acts as a reviewer for various journals and conferences. She has also served as a conference chair, and as a technical committee for numerous international conferences.

Muhammad Fayaz, he is currently perusing Ph.D. in Computer Science. He received MS in Computer Science from SZABIST, Islamabad, Pakistan in 2014. He did MSC from the University of Malakand, KPK, Pakistan in 2011. His areas of interest are NP problems, Approximation Algorithms, Image Processing, and Pattern Recognition. 


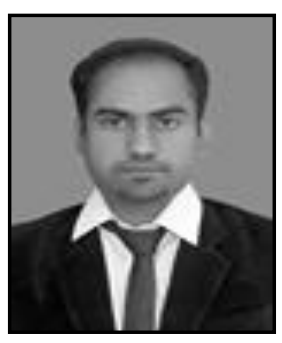

Abdul Salam Shah, he is currently doing specialization in Management Information System (MIS) from Virtual University of Pakistan. He has completed MS degree in Computer Science from SZABIST, Islamabad, Pakistan in 2016. He did his BS degree in Computer Science from Isra University Hyderabad, Sindh Pakistan in 2012. In addition to his degree, he has completed short courses and diploma certificates in Databases, Machine Learning, Artificial Intelligence, Cybercrime, Cybersecurity, Networking, and Software Engineering. He has published articles in various journals of high repute. He is a young professional and he started his career in the Ministry of Planning, Development and Reforms, Islamabad Pakistan. His research area includes Machine Learning, Artificial Intelligence, Digital Image Processing and Data Mining.

Mr. Shah has contributed in a book titled "Research Methodologies; an Islamic perspective," International Islamic University Malaysia, November, 2015. 
International Journal of Bio-Science and Bio-Technology Vol.8, No.6 (2016) 